\title{
Automation of Structural Sizing of Aircraft Concepts Under Static Aeroelastic Constraints
}

\author{
$\mathrm{Wu} \mathrm{Li}^{*}{ }^{*}$ Karl Geiselhart, ${ }^{\dagger}$ Erik Olson, ${ }^{\dagger}$ and Jay Robinson ${ }^{\dagger}$ \\ NASA Langley Research Center, Hampton, Virginia 23681, USA
}

\begin{abstract}
This paper presents an automation process for structural sizing of subsonic and supersonic aircraft concepts under static aeroelastic constraints. The automation process starts with an OpenVSP geometry and ends with a PATRAN plot of a NASTRAN solution for static aeroelastic analysis or optimization. ModelCenter is used to integrate all analysis codes with easy-to-use interfaces. Automation tools are developed to streamline the setup process and avoid user errors. Fuel is distributed by solving an optimization problem to match the center of gravity of aircraft at a specified flight condition. Fuel weights are also automatically attached to the structural model as point masses. All other weights used in FLOPS mission analysis (excluding fuselage and wing structural weights) are automatically attached to or smeared on the structural model. For any given OpenVSP geometry and FLOPS analysis data, a static aeroelastic sizing model for NASTRAN analysis can be generated in a couple of hours. The empirical fuselage and wing structural weights from FLOPS are replaced by structural panel weights from the sized finite-element model. Three supersonic and two subsonic aircraft concepts are used to demonstrate the automation process as a physics-based weight estimation tool for aircraft conceptual design.
\end{abstract}

\section{Nomenclature}

$\mathrm{CG}_{x}=x$-coordinate of center of gravity

$\mathbf{T}_{i} \quad=$ a vector of thickness design variables

$V_{i} \quad=$ volume of the $i$-th specified fuel tank

$W_{i} \quad=$ weight of fuel in the $i$-th specified fuel tank

$x, y, z=$ coordinates of a point in a three-dimensional space

$\beta=$ a percentage parameter for tank storage capacity between 0 and 1

$\delta, \varepsilon \quad=\quad$ error tolerances for alternating projection method

$\lambda=$ a scaling factor $(\geq 1)$ for Young's modulus during structural sizing iterations

$\rho \quad=$ density of fuel

\section{Introduction}

TRADITIONAL weight estimation during conceptual design is often based on empirical data and not applicable to revolutionary or unconventional new aircraft. There is a need to use physics-based weight estimation methods for system-level trade studies of advanced aircraft concepts. However, a relatively long turn-around time (months) of finite-element analysis (FEA) for a complete aircraft hinders the applications of FEA during aircraft conceptual design. Available automation tools for FEA during aircraft conceptual design are only robust enough for individual aircraft components. The generation of finite-element meshes for a complete aircraft is labor-intensive and prone to errors. A recent advance breaks the barrier to automated generation of a fully connected finite-element mesh for the fuselage and wing of an aircraft configuration [1]. The key idea is to generate a geometry model for internal structural components and skins such that any two connected surfaces share a common boundary curve. Such a geometry model is called a finite-element-model-ready (FEM-ready) geometry [1] because it enables automated generation of a fully connected finite-element mesh. This paper continues the work started in Ref. [1] and completes the automation steps from a FEM mesh to a NASTRAN [2] input file for structural sizing under static aeroelastic constraints.

\footnotetext{
* Senior Research Engineer, Aeronautics Systems Analysis Branch

${ }^{\dagger}$ Aerospace Engineer, Aeronautics Systems Analysis Branch
}

American Institute of Aeronautics and Astronautics 
The challenge to building an FEA sizing model from a mesh of quadrilaterals and some triangles is how to develop user interfaces for easy setup of the sizing model and how to manage the inter-related data to generate the correct NASTRAN input file for sizing. The key ideas in this paper for automation of panel thickness sizing of aircraft concepts include: (i) a pattern recognition algorithm to check the connectivity of a generated mesh and reconnect any disconnected nodes once they are identified; (ii) TrimAssist to avoid user errors in defining trim conditions for static aeroelastic analysis; (iii) integration of weight and mission data of Flight Optimization System (FLOPS) [3] in FEA; and (iv) alternating projection for mesh-independent sizing solutions. It is important to point out that the integration interface with FLOPS can be easily adapted to any other aircraft weight estimation and mission analysis tool.

The completed automation process is called ConceptFEA (FEA for aircraft conceptual design) and implemented in ModelCenter [4]. It starts from an OpenVSP [5] geometry and ends with a PATRAN [6] plot of a NASTRAN sizing solution under static aeroelastic constraints. Figure 1 shows a ModelCenter implementation of ConceptFEA.
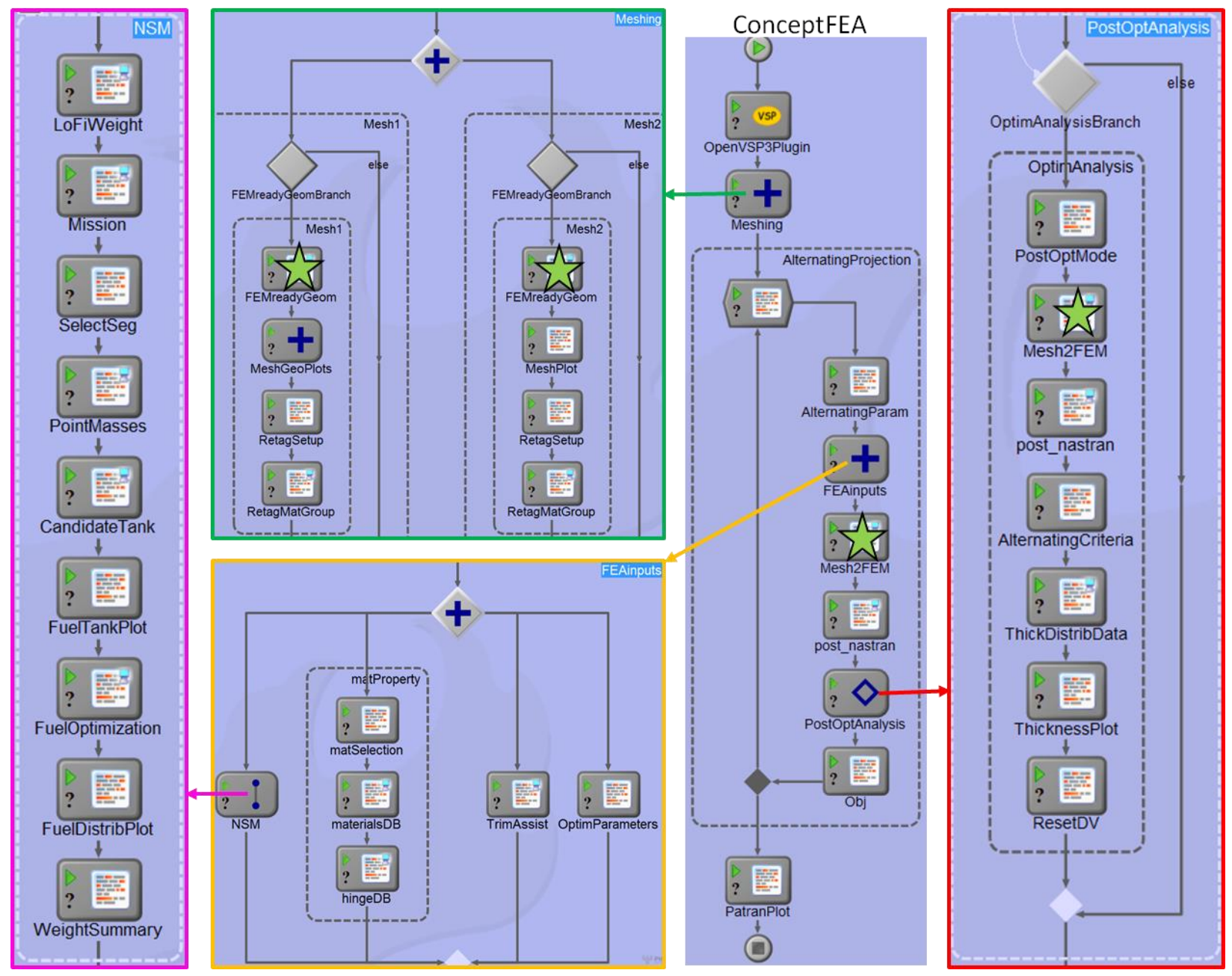

Fig. 1 ModelCenter process for ConceptFEA.

The overall flow of ConceptFEA is shown in the third column, and the colored boxes are expanded views of the assemblies of the analysis components. The key automation components in Fig. 1 are FEMreadyGeom and Mesh2FEM, highlighted by green stars. FEMreadyGeom is the automated meshing process documented in Ref. [1]; it constructs a FEM-ready geometry for the given layout input parameters and then uses PATRAN to generate a fully connected mesh for both the fuselage and wing. Mesh2FEM writes out a structural sizing or analysis model in NASTRAN bulk data format; then it uses NASTRAN to optimize the structure under aeroelastic constraints or to generate aeroelastic analysis results. The alternating projection iteration loop after the "Meshing" assembly in Fig. 1 is used to get mesh-independent sizing solutions for two meshes of different sizes. Here mesh-independent sizing solutions mean that the relative differences between two sets of fuselage and wing weights for two different meshes are within a small tolerance specified by a user (such as 5\%). For sizing of supersonic aircraft concepts under

American Institute of Aeronautics and Astronautics 
displacement constraints, the first sizing solution is most likely mesh-dependent and significantly overestimates the fuselage and wing weights; the alternating projection is an effective method for mesh-independent estimates of fuselage and wing weights.

The other analysis components in Fig. 1 provide a logical break down of the tasks to define a sizing model (such as selection of fuel tank locations by CandidateTank and distribution of fuel by FuelOptimization) and generate plots.

For any given OpenVSP geometry model and FLOPS analysis data, the labor-intensive steps of constructing a sizing model using ConceptFEA are (i) definition of structure layout parameters such as spar, rib, and frame/bulkhead locations, and (ii) selection of $x$-ranges and $y$-range for fuel tank locations in the fuselage and wing, respectively. Usually, these two steps can be completed in a couple of hours for a new aircraft concept.

The structural weight of a wing from a finite-element model is not the same as the as-built weight of a wing. Scaling the estimated structural weight to match the as-built weight is a common practice for physics-based weight estimation methods (e.g., see Refs. [7,8]). However, instead of using a scaling method to estimate as-built wing weight, ConceptFEA uses FEA for structural weight estimates and the regression formulas in FLOPS for non-structural weight estimates. FLOPS non-fuel weight is decomposed into empirical FEM weights, non-structural mass (NSM), and other terms (cf. Fig. 2). Only the empirical FEM weights (the red boxes in Fig. 2) are recalculated by ConceptFEA.

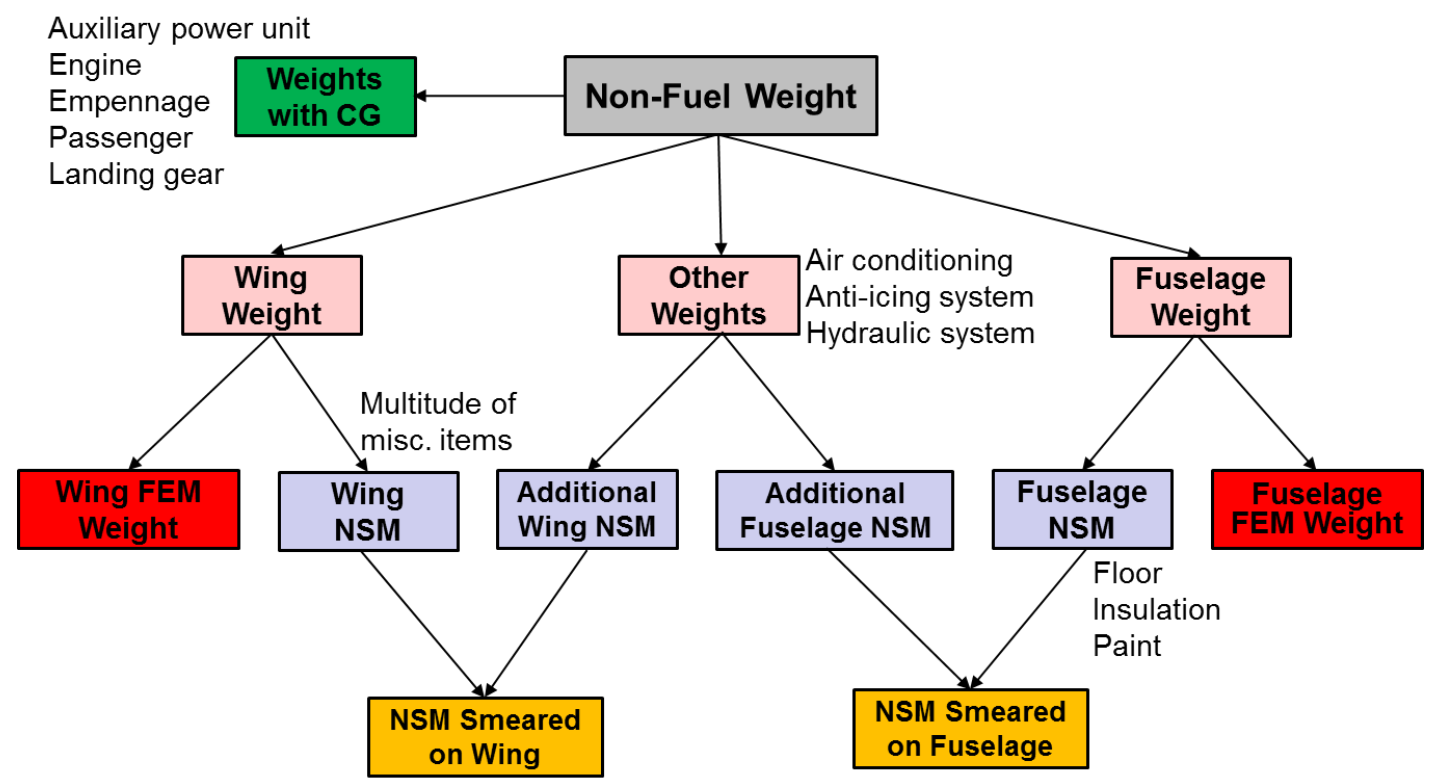

Fig. 2 Decomposition of FLOPS empirical weights.

The verification study of ConceptFEA includes five aircraft concepts. For each aircraft concept, sizing constraints are identified to match [calibrated] FLOPS fuselage and wing weights. Validity of [calibrated] FLOPS weights can be inferred from the validity of identified sizing constraints. For example, if the safety factor for sizing constraints has to be lower than the Federal Aviation Administration (FAA) standard value to match FLOPS weights, then FLOPS weights are considered to be questionable. On the other hand, if the sizing constraints are known, ConceptFEA can generate FEA-based fuselage and wing weights to correct FLOPS (or any other low-fidelity hand-book/semiempirical) weights.

The paper is organized as follows. Section II describes the solution techniques used by ConceptFEA, including a detailed explanation of the decomposition of FLOPS non-fuel weight, fuel distribution, automated attachment of point masses, and alternating projection for mesh-independent solutions. Other automation interfaces and tools are presented in Section III, including post-processing of generated meshes and a detailed explanation of all required inputs. In Section IV, five aircraft concepts are used to demonstrate ConceptFEA as a physics-based weight estimation tool. The concluding remarks are in Section V.

\section{Solution Techniques}

Existing aircraft weight data mostly contain as-built fuselage and wing weights [9], which are not the same as fuselage and wing structural weights from FEA. There is little public information about the structural panel weights for a fuselage and wing. A commonly used approach for as-built weight estimation is to apply a scaling factor to the

American Institute of Aeronautics and Astronautics 
estimated FEM weight [7,8]. In contrast, ConceptFEA uses a combination of the estimated FEM weight and FLOPS regression formulas for non-FEM weights to estimate as-built weight.

FLOPS weight estimation method uses regression formulas for aircraft weight data of 17 transport aircraft and 25 fighter/attack aircraft [10]. The weight formulas are fit to that data using a form that is based on physical characteristics. The newly developed FEA-based weight estimation tool ConceptFEA only aims to improve FLOPS fuselage and wing structural weight estimation using FEA. Subsection II.A has the details of how to use FLOPS nonstructural weights for FEA-based fuselage and wing weight estimation.

Fuel weight distribution is automated by solving a feasibility optimization problem (cf. Subsection II.B). All point masses are automatically attached to the generated finite-element mesh (cf. Subsection II.C).

Structural sizing of a supersonic aircraft configuration is a knowledge-based iterative process that is difficult to be modeled as an optimization problem. In practice, sizing might start with "rigid aerodynamics" and "eventually transition to flexible aerodynamics as the structural design matured" (cf. the paragraph after Fig. 10 in Ref. [11]). Buckling and displacement constraints might be necessary. For example, the FEM of a supersonic N+2 concept was "sized to strength, buckling and manufacturing criteria" and then resized with a tail deformation constraint after discovering excessive tail deformation for one of the pull-up maneuvers (cf. the paragraph after Fig 7 in Ref. [11]).

When using a structural model with all shell elements, buckling analysis is not meaningful. Therefore, displacement constraints are used for supersonic concepts to increase the rigidity of the sized structure. However, displacement constraints lead to a nonlinear weight minimization problem that has many local minima. The relative difference of fuselage/wing weights from two local minima could differ significantly. Such a variation of the estimated weight from structural sizing renders the estimated weight useless. By searching for mesh-independent solutions, inconsistent variations of the estimated weight can be avoided. In this paper, mesh-independent solutions mean that the relative differences between two sets of fuselage and wing weights for two different meshes are within a small tolerance specified by a user. Subsection II.D introduces the alternating projection method to find mesh-independent solutions.

\section{A. Decomposition of FLOPS Non-Fuel Weight}

One significant difference between structural analyses during the early conceptual design and at the later design phases is the vehicle information available for FEA. During the early conceptual design, the geometry shape tends to change drastically and many design details are not finalized. For example, a particular engine might be considered to be a good candidate for the aircraft and an approximate location of the engine is determined, but how the engine is installed on the aircraft is usually determined later in the design process. Therefore, the detailed load path between engine and airframe is usually uncertain during the early conceptual design, as are the fuel tank locations and fuel distributions.

Aircraft weight and mission data are usually generated by systems analysts during early conceptual design. FLOPS weight data (except the structural weights modeled by the FEM) can be included in the FEM to model all required weights carried by the aircraft. For ConceptFEA, FLOPS weight data will be divided into three groups for structural modeling: (i) weights with center of gravity (CG) information (such as landing gear, engine, etc.), (ii) other non-fuel weights without CG (such as hydraulic and electrical systems), and (iii) a lump sum of fuel weight for a specified flight condition.

Figure 2 provides an overview of the decomposition scheme for non-fuel weight. "Wing NSM" is the third term in FLOPS wing weight formula and represents a multitude of miscellaneous items (cf. Eq. (36) in Ref. [10]). FLOPS has no clean decomposition of as-built fuselage weight into structural and non-structural weights. Therefore, the ratio (Wing NSM)/(Wing Skin Area) is also used for fuselage, with two adjustment terms:

$$
(\text { Fuselage NSM })=(\text { Fuselage Skin Area }) \cdot(\text { Wing NSM }) /(\text { Wing Skin Area })+W(\text { skin })+\text { W }(\text { frame })
$$

where W(skin) is the NSM for paint and insulation, and W(frame) is the NSM for other fuselage-specific items not in FEM such as floor weight. W(skin) is smeared on the fuselage skin panels and $\mathrm{W}$ (frame) is smeared on the frames/bulkheads. Here "smearing" means that the weight is uniformly distributed on all involved panels. In theory, one could also use regression formulas to determine what the NSM of fuselage should be. In this paper, Eq. (1) is used by ConceptFEA for computing (Fuselage NSM).

"Other Weights" include all FLOPS weights for physical entities without CG such as air conditioning, anti-icing system, and hydraulic system. Based on FLOPS regression formulas for their influences on wing and fuselage, they are regrouped as "Additional Fuselage NSM" and "Additional Wing NSM" using an analytical formula for the ratio:

$$
\text { (Additional Fuselage NSM) = NSM_Ratio } \cdot(\text { Other Weights) }
$$

American Institute of Aeronautics and Astronautics 


$$
(\text { Additional Wing NSM) }=(1 \text { - NSM_Ratio }) \cdot(\text { Other Weights })
$$

NSM_Ratio $=f($ main gear, nose gear, anti-icing, miscellaneous, tank plumbing, hydraulic, electrical, control surfaces)

where $f(\cdot)$ is an analytical formula of FLOPS weights for eight entities such as the hydraulic system and the electrical system.

Note that Eqs. (2)-(4) provide a decomposition of "Other Weights" in Fig. 2. All the non-structural weights are either attached to FEM as point masses or smeared on the FEM as NSM. So the sizing model carries the same weight as the FLOPS mission analysis model, which is considered to be adequate for FEA-based weight estimation at conceptual level. Here is the decomposition of FLOPS non-fuel weight for FEA.

$$
\begin{aligned}
(\text { FLOPS non-fuel weight })= & \text { (Point masses attached to FEM }) \\
& +(\text { NSM smeared on FEM })+(\text { Empirical FEM weights })
\end{aligned}
$$

Of the three terms in the equation above, only the third term will be replaced by the FEM weights calculated by ConceptFEA. The point masses attached to the FEM are physical items defined by systems analysts and reasonably accurate (the green box in Fig. 2). NSM smeared on FEM are also physical items that are usually defined later in the design process and estimated by FLOPS regression formulas. These smeared weights (the yellow boxes in Fig. 2) are marginally accurate because there is not much physics involved in estimation of these weights. The third term represent empirical estimates of fuselage and wing FEM weights (the red boxes in Fig. 2), which are mainly determined by structural physics and could be incorrectly inferred by the regression formulas. In this paper, these weights are replaced by structural panel weights of the fuselage and wing computed by ConceptFEA using FEA. The fuselage and wing weights generated by ConceptFEA are the following:

$($ Fuselage Weight by ConceptFEA $)=($ Structural Panel Weight of Fuselage $)+($ Fuselage NSM $)$

(Wing Weight by ConceptFEA $)=($ Structural Panel Weight of Wing $)+($ Wing NSM $)$

where (Wing NSM) is the third term in FLOPS wing weight formula (cf. Eq. (36) in Ref. [10]) and (Fuselage NSM) is given in Eq. (1).

An in-depth regression analysis is needed for a more accurate decomposition of NSM for fuselage and wing, but it is out of the scope of this paper.

\section{B. Distribution of Fuel Weight}

FLOPS only provides the total fuel weight for any flight segment of the mission profile, which needs to be distributed for the FEM. There are at least two different approaches to simulate the storage of fuel. One approach is to smear fuel weight on some selected load-bearing structural elements. A more intuitive approach is used by ConceptFEA to include the fuel in the FEM.

The FEM ready-geometry provides a partition of the interior space into closed compartments in the wing. These compartments are considered as possible fuel tank locations. These locations are separated into two groups: the compartments between the front and rear spars as the main group, and the remaining compartments as an auxiliary group. Users can either include or exclude the auxiliary group and use a $y$-range parameter to decide how far in the span-direction fuel can be stored (cf. Fig. 3).

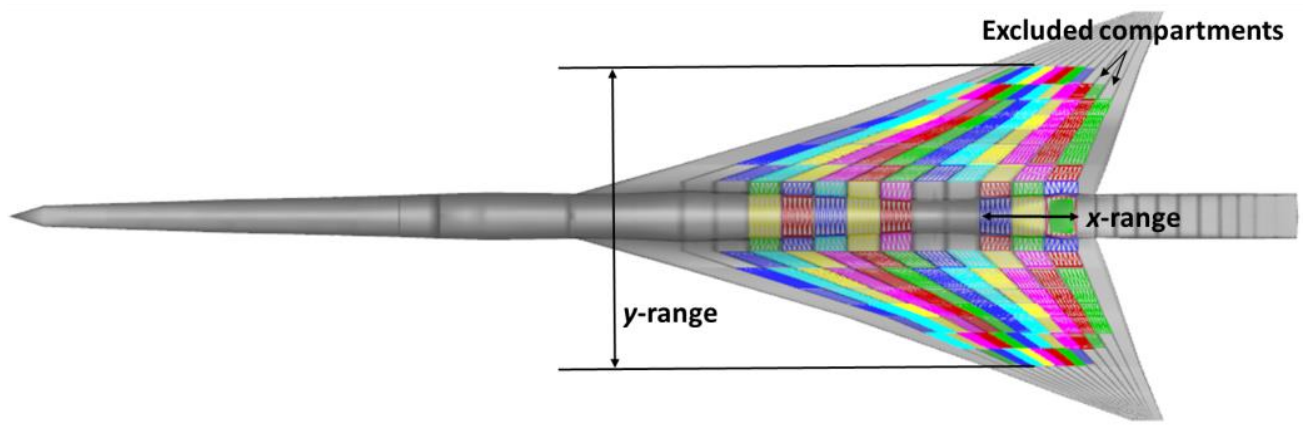

Fig. 3 Specified fuel tank locations.

American Institute of Aeronautics and Astronautics 
For the fuselage, each skin surface in the FEM-ready geometry determines a volume space from one side of the fuselage to the other side, which is conceptually used as a fuel tank in the fuselage. The possible fuel tank locations are determined by a set of intervals in the longitudinal direction: $\left[x_{1, \text { start }}, x_{1, \text { end }}\right],\left[x_{2, \text { start }}, x_{2, \text { end }}\right]$, etc. This simple interface allows a user to easily exclude the locations for storage of main gear, nose gear, cabin, etc., in the fuselage.

The specified tank locations are displayed for visual inspection (cf. Fig. 3). Using the 3D plot, users can find the index of a tank at a particular location and remove it if needed. For example, a few compartments within the specified $y$-range near the rear spar in Fig. 3 are removed from the list of specified fuel tank locations.

FLOPS can generate a $\mathrm{CG}_{x}$ for the zero-fuel weight (ZFW). Along with a target $\mathrm{CG}_{x}$ for the aircraft concept, the target CG for fuel in the longitudinal direction can be calculated as follows:

$$
\left(\text { Target } \mathrm{CG}_{x} \text { for fuel }\right) \cdot(\text { Fuel weight })=\left(\text { Target } \mathrm{CG}_{x} \text { for aircraft }\right) \cdot(\text { Cruise weight })-\left(\mathrm{CG}_{x} \text { for } \mathrm{ZFW}\right) \cdot \mathrm{ZFW}
$$

A feasibility optimization problem can be formulated to find the fuel weights in the specified tanks and match the target fuel $\mathrm{CG}_{x}$ :

$$
\begin{aligned}
& \sum_{i=1}^{n} \mathrm{CG}_{x, i} \cdot W_{i}=\left(\text { Target } \mathrm{CG}_{x} \text { for fuel }\right) \cdot(\text { Fuel weight }) \\
& \sum_{i=1}^{n} W_{i}=\text { (Fuel weight) and } 0 \leq W_{i} \leq \rho \cdot \beta \cdot V_{i} \text { for } 1 \leq i \leq n
\end{aligned}
$$

where $\mathrm{CG}_{x, i}, W_{i}$, and $V_{i}$ are the $x$-coordinate of centroid, fuel weight, and volume for the $i$-th specified tank, respectively, $\rho$ is the fuel density, and $\beta$ is a given storage capacity parameter between 0 and 1 .

\section{Attachment of Point Masses}

Physical entities with CGs in the longitudinal direction and fuel weights from fuel distribution are attached to spars and frames/bulkheads automatically by an attachment algorithm. For a point mass attached to the wing, the attachment algorithm finds the compartment that is closest to the point mass and identifies all the corner points of the compartment; then uses NASTRAN RBE3 elements to attach the point mass to the corner points. For a point mass attached to the fuselage, the attachment algorithm finds the two frame/bulkhead locations closest to the point mass and identifies the four grid points on the intersection of each frame/bulkhead and the symmetry plane $y=0$; then uses NASTRAN RBE3 elements to attach the point mass to the identified grid points on the symmetry plane. Figure 4 shows how point masses are attached to the finite-element mesh.

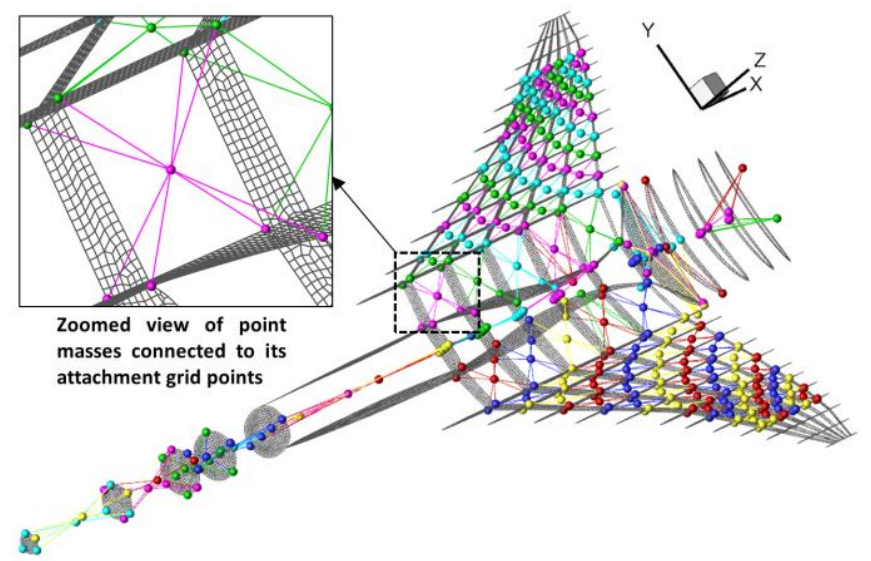

Fig. 4 Attachment of point masses to finite-element mesh.

\section{Alternating Projection for Mesh-Independent Solutions}

One desirable feature of any FEA-based weight estimation tool is that the estimated weight is independent of the finite-element mesh used. That is, it is desirable to obtain mesh-independent solutions. There is no rigorous definition 
for mesh-independent solutions. In this paper, the estimated weights are considered to be mesh-independent if the two set of weights of the sized structure models for two meshes of "significantly different sizes" differ by less than 5\%. The qualitative quantifier "significantly different sizes" means the numbers of quadrilaterals in two meshes differ by more than $20 \%$.

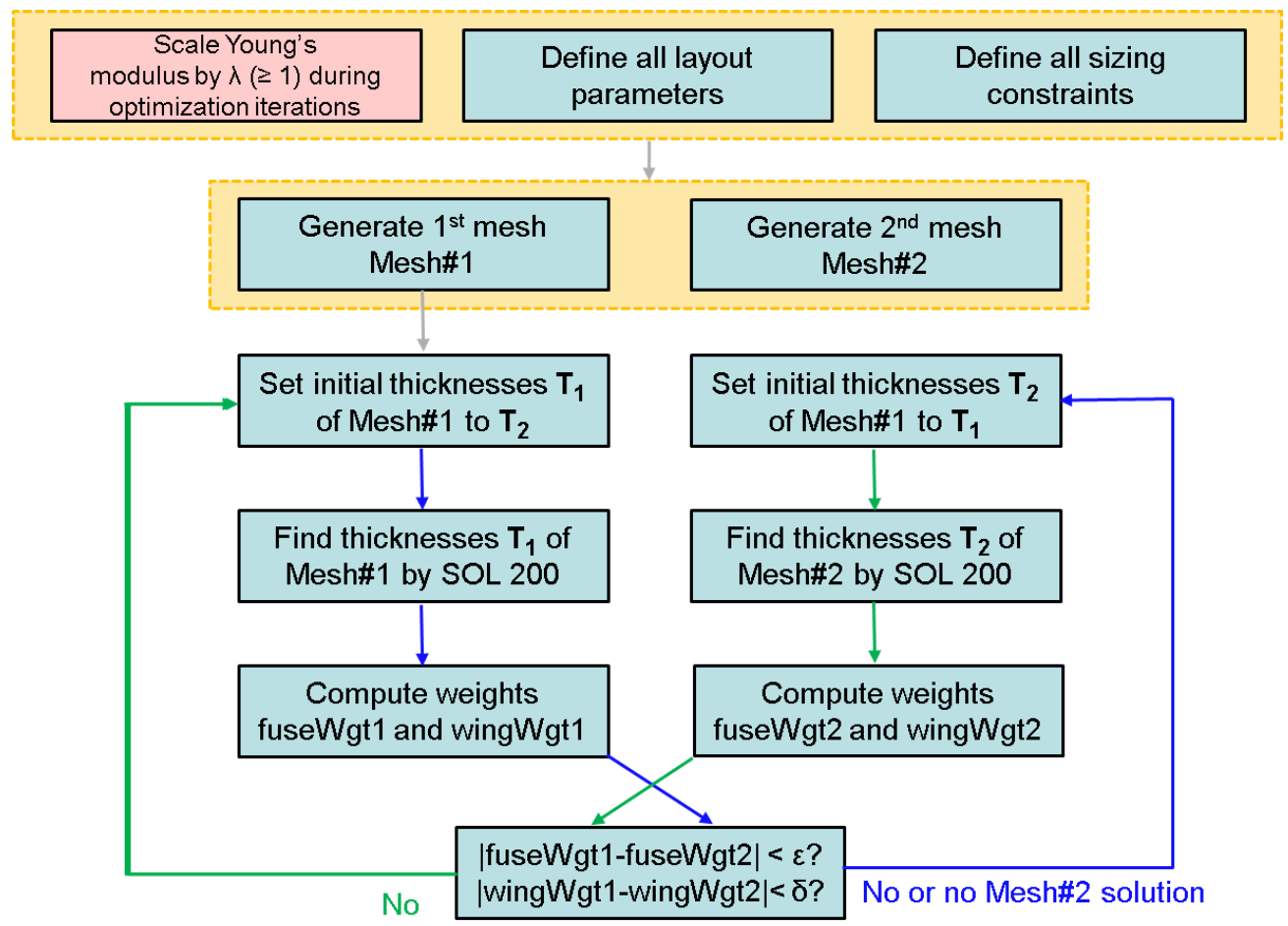

Fig. 5 Alternating projection algorithm for two meshes.

A flowchart for the alternating projection used by ConceptFEA is shown in Fig. 5. Once all layout parameters and sizing constraints are defined, two meshes of different sizes are generated. All thickness design variables of $\mathbf{T}_{2}$ are set to a user-specified value and used as an initial guess for Mesh\#1. The optimal thickness vector $\mathbf{T}_{1}$ for Mesh\#1 is computed using NASTRAN SOL 200. Then $\mathbf{T}_{1}$ is used as the initial thicknesses for Mesh\#2 and the optimal thickness vector $\mathbf{T}_{2}$ is calculated for Mesh\#2 using NASTRAN SOL 200. Now there are two sets of fuselage and wing weights. If the differences are not within the specified tolerances, then $\mathbf{T}_{2}$ is used as the initial thicknesses for Mesh\#1, the optimal thickness vector $\mathbf{T}_{1}$ is recalculated for Mesh\#1, and the two sets of fuselage and wing weights are compared again. The iteration continues until the differences are within the specified error tolerances. To avoid aeroelastic analysis failure, it may be necessary to scale Young's modulus for all materials by $\lambda(\geq 1)$ during optimization iterations for supersonic concepts. The scaling does not affect stress constraints, but displacement constraints have to be adjusted for the scaling.

Because displacement constraints are highly nonlinear with respect to thickness design variables, the corresponding optimization problem tends to have many local minima. The alternating projection method is also very effective to move away from a local minimum toward a global minimum.

\section{Automation for Construction of Sizing Model}

For given required inputs, ConceptFEA automatically generates the NASTRAN inputs in bulk data format for thickness design optimization or static aeroelastic analysis. This section describes the interface tools used to automate the construction of a sizing model.

Subsection III.A explains all of the required inputs needed to generate a sizing model. Even though the mesh generation is automated using FEM-ready geometry and PATRAN, there is a need for post-processing of the generated mesh, which is presented in Subsection III.B. Subsection III.C introduces TrimAssist, an interface tool to alleviate a novice user's frustration of defining all the trim variables properly. Automated aero panel generation and aerostructure coupling are briefly described in Subsection III.D. In summary, once the required inputs are provided, ConceptFEA generates a sizing model automatically and robustly. The generated sizing model is for weight

American Institute of Aeronautics and Astronautics 
minimization with respect to thickness design variables under stress and other constraints for a variety of trim conditions.

\section{A. Required Inputs}

An OpenVSP geometry and the related FLOPS analysis data are a prerequisite for ConceptFEA structural sizing. ModelCenter makes ConceptFEA very easy to use. Figure 6 shows all required inputs for a basic sizing run.

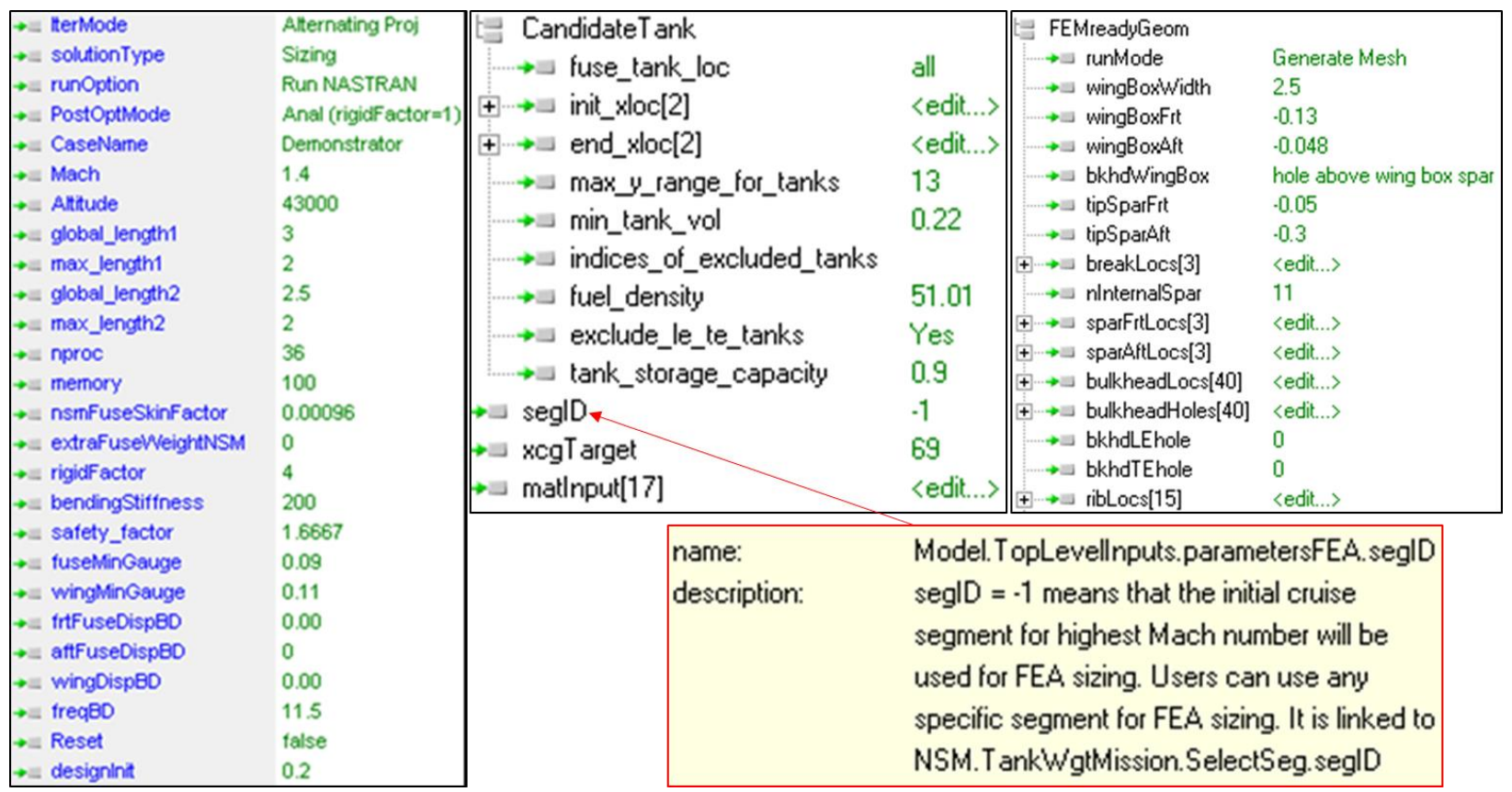

Fig. 6 All required inputs for ConceptFEA implemented in ModelCenter.

Figure 6 intends to provide an overview of the amount of information that must be specified by a user for a successful sizing run. Each input of ConceptFEA is annotated with some comments on its usage. The lower right box of Fig. 6 is from a screen shot of the pop-up text when hovering the mouse cursor over the input variable "segID".

The inputs in the upper right box of Fig. 6 contains the layout parameters to define spars, ribs, and frames/bulkheads (cf. Ref. [1] for more details on the layout definition). The inputs under CandidateTank in the upper middle box of Fig. 6 define the specified fuel tank locations, fuel density, and tank storage capacity ( $\beta$ in Eq. (10)). The fuel distribution optimization is determined by "xcgTarget" in the upper middle box of Fig. 6, which is "Target $\mathrm{CG}_{x}$ for aircraft" in Eq. (8).

The generated mesh is partitioned into 16 default material groups [1]. There is an option to create additional material groups (using RetagMatGroup inside "Meshing" assembly [green box] in Fig. 1). In Fig. 6, there are 17 predefined material groups for the generated mesh and the material property assignment is simplified to define 17 entries of the array matInput[] in the upper middle box of Fig. 6. Each entry of matInput[] can be either the name or index of a material in a material database file [1].

The left box in Fig. 6 contains all the remaining parameters that require manual inputs from a user. The first 4 parameters are the same for structural sizing. "CaseName" is used to label the saved optimization results. "Mach" and "Altitude" are used to define the trim conditions for $2.5 \mathrm{G}$ pull-up, $1 \mathrm{G}$ cruise, and $-0.5 \mathrm{G}$ push-over maneuvers. The next 4 inputs are length parameters for the PATRAN meshing code Paver. They are used to generate two meshes of different sizes. In general, several trials are required to generate two meshes of desired sizes.

Parameters "nproc" and "memory" are used to request the number of cores and maximum memory size (in GB) on a Linux server that runs NASTRAN. NASTRAN allows parallel execution, but the total wall time depends more on the core speed than the number of cores [12].

Parameter "nsmFuseSkinFactor" multiplied by the total fuselage skin area is W(skin) in Eq. (1), while parameter "extraFuseWeightNSM" is W(frame) in Eq. (1). There is some guideline on the value of "nsmFuseSkinFactor" based on the known weight information for paints and insulation materials.

Scaling of Young's modulus for all materials is determined by "rigidFactor" ( $\lambda$ in Fig. 5). To avoid local vibration modes in NASTRAN modal analysis, "bendingStiffness" is used to artificially increase the bending stiffness of non-

American Institute of Aeronautics and Astronautics 
skin panels. It has almost no effect on structural sizing of subsonic concepts and marginal effects on structural sizing of supersonic concepts.

The remaining parameters in the left box of Fig. 6 define the bounds for thickness design variables and constraints. Because the yield stress for each material is stored in the material database file, "safety_factor" sets the stress upper bound for each panel to (yield stress)/safety_factor. Parameters "fuseMinGauge" and "wingMinGauge" define the lower bounds for thickness design variables of fuselage and wing panels, respectively. The next 3 parameters define the maximum displacement limits for wing tip, fuselage nose, and fuselage tail, respectively. A positive lower bound "feqBD" for the lowest frequency will increase structural rigidity and weight simultaneously. Setting a constraint bound to zero will remove the corresponding constraint from the sizing model. Thickness design variables are automatically determined by the structural layout. All panels in each surface in FEM-ready geometry model share one thickness design variable (cf. Ref. [1] for more details). By setting "Reset" in the left box of Fig. 6 to true, ConceptFEA will automatically reset all initial design variables to the value specified by "designInit" for NASTRAN SOL 200 run.

\section{B. Verification and Quality Improvement of Finite-Element Meshes}

The automated meshing process developed in Ref. [0] is further tested with more configurations. As the geometry shape becomes more complicated (such as a concave downward upper surface of the aft fuselage), it requires trialand-error to use a proper equivalence tolerance for PATRAN to generate a fully connected finite-element mesh. Such an interactive meshing process is time consuming and prone to user errors. A more desirable solution is a numerical algorithm that can detect whether the generated finite-element mesh is fully connected or not. While such an algorithm (to check watertight connectivity of faces) is available for computational fluid dynamic meshes, there is no counterpart for finite-element meshes. For a finite-element mesh, one can use PATRAN to identify free edges of a generated mesh, but a user has to visually inspect the free edges to determine whether any of the free edges represents an unexpected disconnection (or error) in the underlying mesh. A pattern recognition algorithm based on FEM-ready geometry is developed to check the connectivity of a generated mesh and reconnect any disconnected nodes once they are identified. This algorithm has been successfully applied to fix PATRAN meshes with unexpected connection errors.

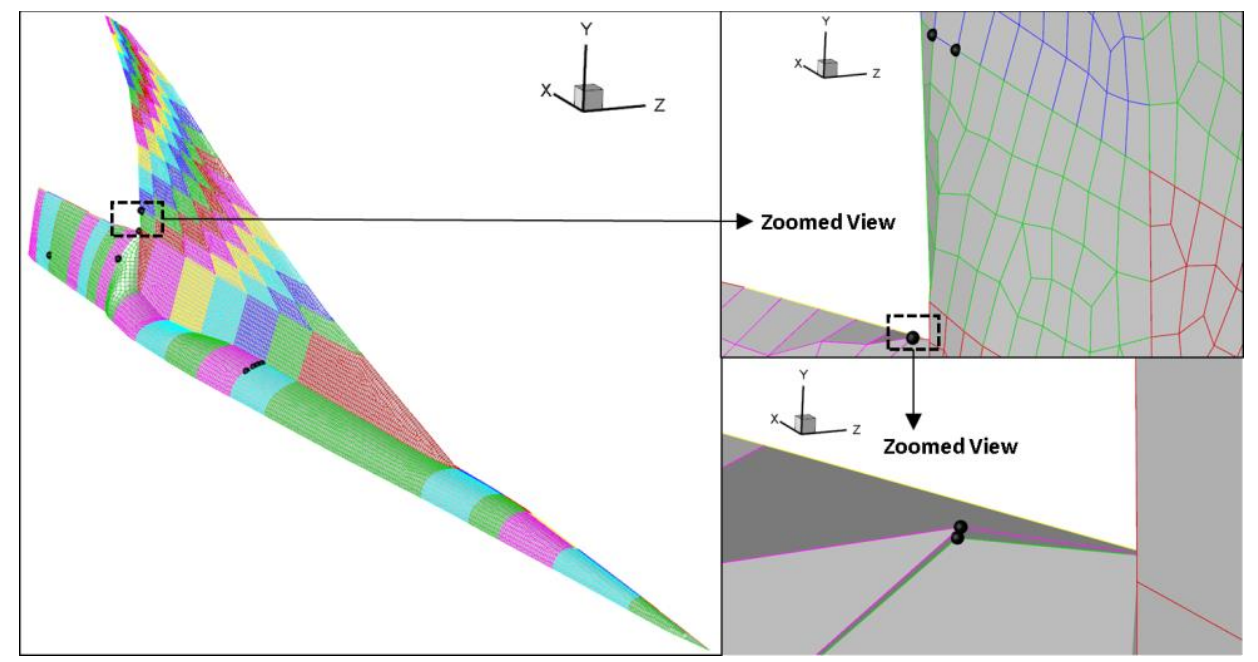

Fig. 7 Detection of disconnected nodes in finite-element mesh.

Figure 7 shows a high-quality PATRAN mesh with 9 pairs of disconnected nodes. The maximum error for the disconnected node pairs is $0.021 \mathrm{ft}$ for a vehicle of $240 \mathrm{ft}$ long. Each pair of disconnected nodes resembles a "popped rivet" in structural construction. NASTRAN will generate a sized structure for this mesh; and users will not know that there are connection errors in the model without a time-consuming inspection of the mesh. Of course, a proper choice of equivalence tolerance for the PATRAN mesh merging algorithm will result in the correct mesh, but it is not easy to know a priori what the tolerance should be. The newly developed algorithm will find all disconnected node pairs and reconnect them to form a fully connected mesh.

Another issue is that the maximum warp angle and minimum/maximum angle for all panels affect the numerical accuracy of aeroelastic analysis. A post-meshing algorithm is implemented to split a quadrilateral panel into two triangular ones if either the warp angle is greater than $45 \mathrm{deg}$ or the maximum angle is greater than $175 \mathrm{deg}$; moreover, any sliver triangle (with the minimum angle less than $5 \mathrm{deg}$ ) is removed by collapsing the two close-by nodes into one.

American Institute of Aeronautics and Astronautics 
These two algorithms ensure that the generated mesh is fully connected and the mesh quality is sufficient for aeroelastic analysis at the conceptual design level. Any meshing issue will be captured automatically and a plot shows the problem region on the mesh. The plot helps a user to understand what the meshing problem is (such as a sharp turn on a fuselage cross section) and how to modify the geometry to avoid it.

\section{TrimAssist for Easy Setup of Trim Conditions}

Depending on the trim surfaces involved and the boundary conditions for static aeroelastic analysis (using symmetry or a free flight condition), it can be difficult for a casual user of FEA to set up the trim parameters correctly. However, it is feasible to put all the rules for setting up the trim parameters into an algorithm and make the setup process intuitive for novice users. The developed tool is called TrimAssist, which is a Modelcenter analysis component and modifies the inputs needed for an underlying script wrapper upon execution. Based on what the required maneuver condition is, TrimAssist will only expose the inputs required to define the trim condition. The underlying script wrapper will determine all other trim input parameters depending on the trim surfaces available and the specified boundary condition.

\begin{tabular}{|c|c|c|c|}
\hline \multirow{2}{*}{\multicolumn{2}{|c|}{$\begin{array}{l}\text { TrimAssist } \\
\text {. t }{ }^{2} \text { Case1 }\end{array}$}} & \multicolumn{2}{|l|}{ Trimdssist } \\
\hline & & 讯 Case1 & \\
\hline$\rightarrow$ Title & Trim analysis for case 1 & 俴 Case2 & \\
\hline$\rightarrow$ Mach & 1.7 & $\rightarrow$ Title & Trim analysis for case2 \\
\hline$\rightarrow$ Altitude & 41016 & $\rightarrow \rightarrow$ Mach & 1.7 \\
\hline$\rightarrow$ URDD3 & 2.5 & $\rightarrow=$ Altitude & 46016 \\
\hline$\rightarrow \mathrm{PITCH}$ & 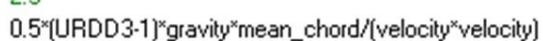 & $\rightarrow \square$ & 0 \\
\hline$\rightarrow$ TrimMode & pullup/pushover/cruise & $\rightarrow=$ URDD3 & 1.5 \\
\hline$\longrightarrow$ BoundaryCondition & Free Flight & $\rightarrow=\mathrm{ROLL}$ & 0.02 \\
\hline$\rightarrow \mathrm{Q}$ & 0 & $\rightarrow$ TrimMode & steady roll \\
\hline$\rightarrow A_{0} A$ & free & $\rightarrow$ BoundaryCondition & Free Flight \\
\hline$\leftrightarrow$ SIDES & 0.0 & $\rightarrow A_{\alpha} \mathrm{A}$ & free \\
\hline$\Rightarrow$ ROLL & 0.0 & $\rightarrow$ SIDES & 0.0 \\
\hline$\leftrightarrow \mathrm{YAW}$ & 0.0 & $\rightarrow \mathrm{PITCH}$ & 0.0 \\
\hline$\longrightarrow$ URDD1 & free & $\rightarrow$ YAW & 0.0 \\
\hline$\rightarrow$ URDD2 & free & $\rightarrow$ URDD1 & free \\
\hline$\rightarrow$ URDD4 & free & $\rightarrow$ URDD2 & free \\
\hline$\longrightarrow$ URDD5 & $\begin{array}{l}\text { rree } \\
0.0\end{array}$ & $\rightarrow$ URDD4 & 0.0 \\
\hline$\longrightarrow$ URDD6 & free & $\rightarrow$ URDD5 & 0.0 \\
\hline$\longrightarrow$ htail_angle & liee & $\rightarrow$ URDD6 & free \\
\hline$\rightarrow$ vtail_angle & riee & $\longrightarrow$ htail_angle & free \\
\hline$\rightarrow$ ailout_angle & not used & $\rightarrow$ vail_angle & not used \\
\hline $\begin{aligned} \rightarrow & \rightarrow \text { allout_angle } \\
\rightarrow & \text { ailin angle }\end{aligned}$ & not used & $\rightarrow$ ailout_angle & not used \\
\hline$\leftrightarrow$ ailin_angle & not used & $\rightarrow$ ailin_angle & not used \\
\hline
\end{tabular}

Fig. 8 User Interface for input parameters for trim analysis.

Figure 8 shows the user interfaces for two different trim cases: pull up and steady roll. A user specifies the number of trim cases to be considered. A pull-down menu allows the selection of a maneuver condition for each case. Then, by executing "Invoking Component Method" in ModelCenter, the required input parameters for each case will be activated and the remaining parameters will be determined based on the rules for trim analysis. For example, for the pull up maneuver in Case 1, only Mach number, altitude, and load multiple in the $z$-direction (URDD3 in NASTRAN) are required. The pitch rate ("PITCH" parameter in Case 1) is determined by the standard formula shown in the interface. For the steady roll maneuver in Case 2, only Mach number, altitude or dynamic pressure (Q), URDD3, and ROLL (roll rate) are required. However, when this component is executed to define the trim parameters, an error message will be generated: "Must define an aileron for steady roll!" In other words, TrimAssist will ensure that the defined trim condition is feasible for analysis. All the information about existing aerodynamic surfaces are automatically passed from FEMreadyGeom to TrimAssist in Fig. 1. For example, if a vertical tail is included in the original geometry model, then vtail_angle will be set to 0.0 deg for pull-up maneuver instead of "not used". Of course, an expert user can ignore the outputs from TrimAssist and manually define all input parameters for any trim analysis.

\section{Aero-Structure Coupling}

For the current implementation of ConceptFEA, only flat aero panels are used for NASTRAN aerodynamic analysis. These aero panels are generated based on two input parameters. One specifies the width of aero panels and another specifies the maximum length of aero panels. Some rules are used to ensure that the minimum angle of any aero panel is not too small. Aero-structure coupling is implemented via the NASTRAN SPLINE1 entity. Each aero panel is mapped to grid points of load-bearing elements in its "projected region of influence," which is a small

American Institute of Aeronautics and Astronautics 
neighborhood of the region under the normal projection of the aero panel. PATRAN plot can be used to check the aerodynamic model (cf. Fig. 9).

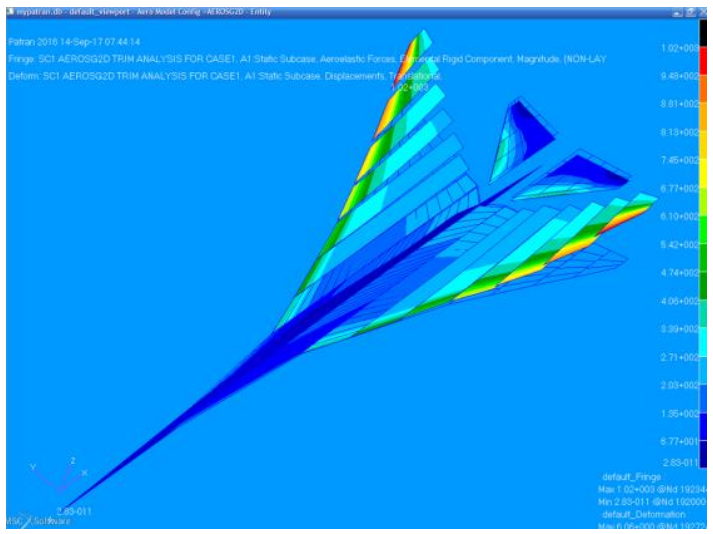

Fig. 9 Aeroelastic forces for $2.5 \mathrm{G}$ pull-up maneuver.

\section{Numerical Examples}

For a given OpenVSP geometry, once FLOPS weight and mission data are available, a sizing model can be defined easily in a couple of hours using ConceptFEA. If the sizing constraints are known, it usually takes less than 12 hours to complete the alternating projection run for mesh-independent estimates of fuselage and wing weights. The sizing goal is not to optimize the structural layout for the lowest structural weight; so a standard layout topology is acceptable. The challenge for sizing an unconventional vehicle is that the sizing constraints might be unknown at the conceptual design phase. The next best option is to reverse-engineer the sizing constraints to match FLOPS weight estimates. If the resulting sizing constraints are considered to be invalid, then FLOPS weights are questionable; otherwise, FLOPS weights are credible. In other words, ConceptFEA is developed as an enhancement tool for FLOPS empirical weight estimation. When the sizing constraints are known, ConceptFEA can also provide the corrections to FLOPS fuselage and wing weight estimates. The integration of FLOPS regression formulas in ConceptFEA (cf. Subsection II.A) eliminates the need of applying empirical scaling factors to the structural weights for estimations of as-built weights.

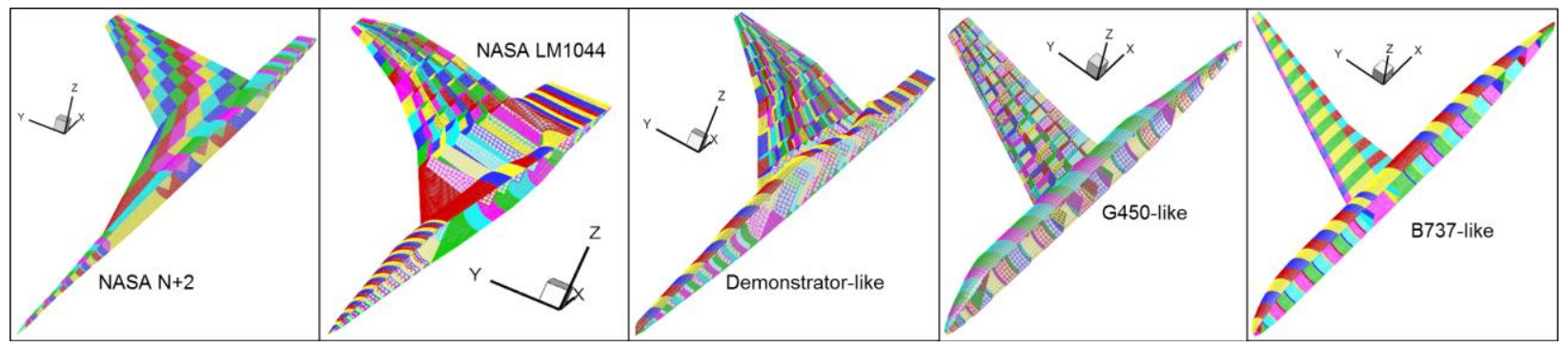

Fig. 10 Structural layouts of five aircraft concepts.

Five aircraft concepts (shown in Fig. 10) are used to demonstrate ConceptFEA as a physics-based weight estimation tool for aircraft conceptual design. The NASA N+2 and NASA LM1044 layouts are based on some empirical data about spar, rib, and frame spacing. The demonstrator-like layout mimics the layout of a more realistic structure model for a preliminary design study of a supersonic demonstrator. The G450-like layout is based on a cutaway drawing of Gulfstream G450, while the B737-like layout is based on a cutaway drawing of Boeing 737. All meshes for this paper are generated using the PATRAN meshing code Paver. Post-meshing algorithms (cf. Subsection III.B) ensure that all panels in a mesh are fully connected, the minimum angle for all panels is no less than 5 deg, and the maximum warp angle is no more than $45 \mathrm{deg}$. Two meshes are used for each concept for mesh-independent solutions. The first mesh has at least 10,000 quadrilaterals and the second mesh has at least $20 \%$ more quadrilaterals than the first. The $2.5 \mathrm{G}$ pull-up, $1 \mathrm{G}$ cruise, and $-0.5 \mathrm{G}$ push-over maneuver conditions are used for aeroelastic sizing. Initial thickness values for all shell elements are 0.2 inches. For each concept, the fuselage and wing weights generated by ConceptFEA are completely determined by safety factor, minimum gauges, displacement limits, and lower bound for minimum frequency. There were multiple trial runs before the desired constraints are identified for each concept to match FLOPS weights. Only the results for the desired constraints are included in the following five subsections.

American Institute of Aeronautics and Astronautics 
For this verification study, the same maneuver conditions are used for sizing supersonic and subsonic concepts. In reality, more maneuver conditions would be used for each concept. Moreover, except for the demonstrator-like concept, more realistic material assignments must be specified for sizing of these concepts.

\section{A. NASA N+2 Supersonic Concept}

NASA N+2 supersonic concept is a low-boom supersonic transport concept envisioned to enter service in 2035. It has a cruise Mach of 1.6, a cruise altitude of 54,000 ft, a cruise weight of 321,703 lbs, a ZFW of 146,115 lbs, and a range of 4,097 nm. Composite material technologies are used for FLOPS weight estimates. The sizing model for FEA uses an aluminum material for all panels. The layout definition leads to 438 thickness design variables. Two meshes of 23,470 and 40,376 quadrilaterals are used for the alternating projection method. To avoid aeroelastic analysis failure, $\lambda=4$ is used during optimization iterations. After setting safety factor $=1.43$, min fuselage gauge $=0.13$ inches, min wing gauge $=0.07$ inches, $\max$ wing displacement $\leq 2.5 \mathrm{ft}$, max front fuse displacement $\leq 0.8 \mathrm{ft}$, and max aft fuse displacement $\leq 0.4 \mathrm{ft}$, the FEA-based fuselage and wing weights match FLOPS fuselage and wing weights with relative errors less than $1.0 \%$ (cf. Fig. 11).

\begin{tabular}{|c|c|c|}
\hline NASA N+2 & Fuselage & Wing \\
\hline 23,470 Quads & $22,274 \mathrm{lbs}$ & $32,347 \mathrm{lbs}$ \\
\hline 40,376 Quads & $21,916 \mathrm{lbs}$ & $31,910 \mathrm{lbs}$ \\
\hline FLOPS Weights & $22,116 \mathrm{lbs}$ & $32,055 \mathrm{lbs}$ \\
\hline
\end{tabular}

Fig. 11 Replication of FLOPS weights for NASA N+2 concept using mesh-independent solutions.

For this concept, the alternating projection method is absolutely needed because the initial thickness values of 0.2 inches lead to a local minimum, resulting in estimated fuselage and wing weights at least $20 \%$ heavier than the weights from the converged mesh-independent solutions. Figure 12 shows the convergence history for the alternating projection.

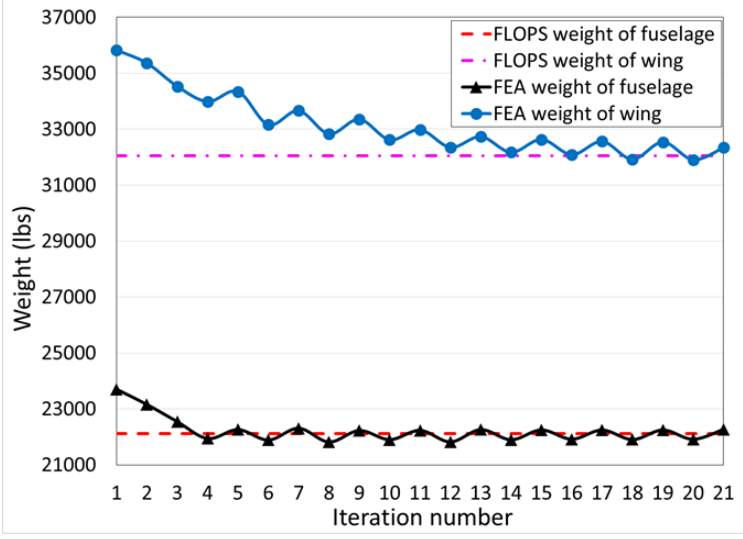

Fig. 12 Alternating projection iteration for NASA N+2 concept to replicate FLOPS weights.

The max displacements for two FEA solutions at $2.5 \mathrm{G}$ trim condition are about $6 \mathrm{ft}$ when analyzed without scaling of Young's modulus (i.e., $\lambda=1$ ), which is higher than the displacement limit of $2.5 \mathrm{ft}$ because the displacement constraints are defined for the scaled problem.

Because a safety factor of 1.43 is less than the FAA standard value of 1.5, one could infer that FLOPS weights underestimate the structural weights for fuselage and wing if the aluminum material is used. This is not unexpected because FLOPS weights account for some weight reduction from future composite material technologies.

\section{B. NASA LM1044 Supersonic Concept}

NASA LM1044 supersonic concept is also a low-boom supersonic transport concept envisioned to enter service in 2035. It has a cruise Mach of 1.7, a cruise altitude of 46,000 ft, a cruise weight of 304,697 lbs, a ZFW of 152,080 $\mathrm{lbs}$, and a range of 5,434 nm. This concept is derived from Lockheed Martin N+2 concept in Ref. [13]. Composite material technologies are used for FLOPS weight estimates. The sizing model for FEA uses an aluminum material for all panels. The layout definition leads to 513 thickness design variables. Two meshes of 17,725 and 22,565 quadrilaterals are used for the alternating projection method. To avoid aeroelastic analysis failure, $\lambda=4$ is used during

American Institute of Aeronautics and Astronautics 
optimization iterations. The sizing constraints are exactly the same as those for NASA N+2, except min fuselage gauge is 0.09 inches instead of 0.13 inches. FEA-based fuselage and wing weights match FLOPS fuselage and wing weights with relative errors less than $1.7 \%$ and $3.5 \%$, respectively (cf. Fig. 13).

\begin{tabular}{|c|c|c|}
\hline NASA LM1044 & Fuselage & Wing \\
\hline 17,725 Quads & $22,099 \mathrm{lbs}$ & $30,829 \mathrm{lbs}$ \\
\hline 22,565 Quads & $22,407 \mathrm{lbs}$ & $31,164 \mathrm{lbs}$ \\
\hline FLOPS Weights & $22,471 \mathrm{lbs}$ & $30,144 \mathrm{lbs}$ \\
$\begin{array}{c}\text { Span }=84 \mathrm{ft} \\
\text { Length }=240 \mathrm{ft}\end{array}$ \\
\hline
\end{tabular}

Fig. 13 Replication of FLOPS weights for NASA LM1044 concept using mesh-independent solutions.

The alternating projection method is also needed for this concept. Figure 14 shows the convergence history for the alternating projection. The max displacements for two FEA solutions at 2.5G trim condition are about $5.7 \mathrm{ft}$ when analyzed without scaling of Young's modulus (i.e., $\lambda=1$ ). Similarly, one could consider that FLOPS weights underestimate the structural weights for fuselage and wing if the aluminum material is used.

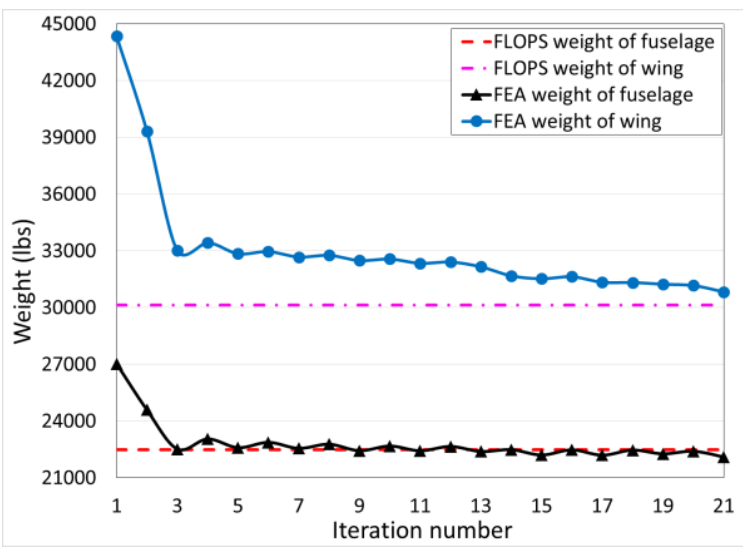

Fig. 14 Alternating projection iteration for NASA LM1044 concept to replicate FLOPS weights.

\section{Supersonic Demonstrator Concept}

The demonstrator-like concept is a low-boom supersonic concept to demonstrate the feasibility of supersonic flight over land with acceptable sonic boom loudness. It has a cruise Mach of 1.4, a cruise altitude of 53,000 ft, a cruise weight of 20,422 lbs, a ZFW of 15,822 lbs, and a range of 1,168 nm. FLOPS weights are calibrated using FEA-based weight estimates from a preliminary sizing study by FEA experts. The sizing model generated by ConceptFEA uses four different materials: two kinds of aluminum, titanium, and a material resembling a composite. The material property assignments mimic the detailed structure model generated by FEA experts. The layout definition leads to 765 thickness design variables. Two meshes of 22,859 and 48,866 quadrilaterals are used for the alternating projection method. To avoid aeroelastic analysis failure, $\lambda=4$ is used during optimization iterations. After setting safety factor $=1.67$, min fuselage gauge $=0.09$ inches, min wing gauge $=0.11$ inches, and min frequency $\geq 11.5$, the FEA-based fuselage and wing weights match the calibrated FLOPS fuselage and wing weights with relative errors less than $1.0 \%$ and $2.1 \%$, respectively (cf. Fig. 15). The max displacements for two FEA solutions at $2.5 \mathrm{G}$ trim condition are about $0.57 \mathrm{ft}$ when analyzed without scaling of Young's modulus (i.e., $\lambda=1$ ). For this sizing problem, all weights generated during alternating projection iterations differ by less than 5\%; so the alternating projection iteration is not necessary. Because the safety factor of 1.67 exceeds the FAA standard value of 1.5, the calibrated FLOPS weights are considered to be credible.

\begin{tabular}{|c|c|c|}
\hline Demonstrator & Fuselage & Wing \\
\hline 22,859 Quads & $3,048 \mathrm{lbs}$ & $3,398 \mathrm{lbs}$ \\
\hline 48,866 Quads & $3,020 \mathrm{lbs}$ & $3,417 \mathrm{lbs}$ \\
\hline Calibrated FLOPS Weights & $3,019 \mathrm{lbs}$ & $3,353 \mathrm{lbs}$ \\
$\begin{array}{c}\text { Span }=29.4 \mathrm{ft} \\
\text { Length }=92.6 \mathrm{ft}\end{array}$ \\
\hline
\end{tabular}

Fig. 15 Replication of calibrated FLOPS weights using mesh-independent solutions (using $\lambda=4$ ).

American Institute of Aeronautics and Astronautics 
To confirm that artificial scaling does not affect the estimated weights, the structure is resized without artificial scaling during the optimization iterations (i.e., $\lambda=1$ ). Without scaling of Young's modulus, NASTRAN modal analysis shows that the previously sized structure has the minimum frequency of 5.73. Replacing the previous lower bound of 11.5 for the frequency constraint by 5.73, the resulting weights for the newly sized structure are almost identical to those in Fig. 15 and the relative matching errors are less than 1.0\% (cf. Fig. 16). The max displacements for two FEA solutions at $2.5 \mathrm{G}$ trim condition are about $0.56 \mathrm{ft}$, similar to the previous analysis result of $0.57 \mathrm{ft}$.

\begin{tabular}{|c|c|c|}
\hline Demonstrator & Fuselage & Wing \\
\hline 22,859 Quads & $3,046 \mathrm{lbs}$ & $3,375 \mathrm{lbs}$ \\
\hline 48,866 Quads & $3,043 \mathrm{lbs}$ & $3,368 \mathrm{lbs}$ \\
\hline Calibrated FLOPS Weights & $3,019 \mathrm{lbs}$ & $3,353 \mathrm{lbs}$ \\
\hline
\end{tabular}

Fig. 16 Replication of calibrated FLOPS weights using mesh-independent solutions (using $\lambda=1$ ).

In general, it is difficult to visualize small variations between two arrays of 765 thickness design variables. So a histogram analysis of thickness variables is performed for the optimized thickness design variables of two different meshes. The resulting histograms in Fig. 17 show remarkable statistical similarity of the two sized structures.

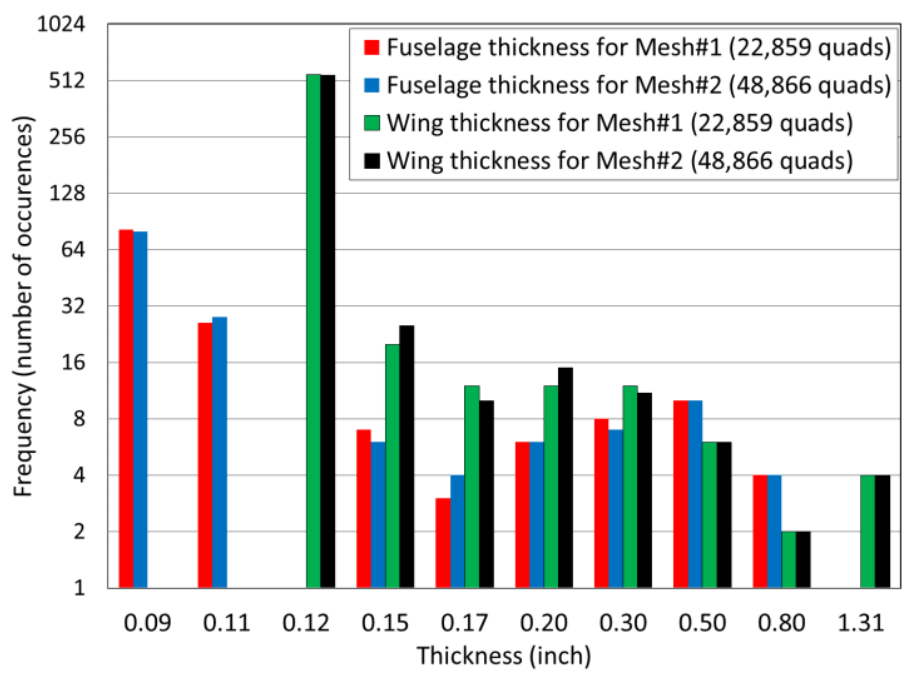

Fig. 17 Comparison of histograms of optimal thickness variables for two meshes.

\section{G450-like Subsonic Concept}

The fourth example is a G450-like subsonic concept. Its empty weight is similar to Gulfstream G450's empty weight and its mission profile is typical of a subsonic business jet. This concept has a cruise Mach of 0.8 , a cruise altitude of $41,000 \mathrm{ft}$, a cruise weight of 73,861 lbs, a ZFW of 43,351 lbs, and a range of 5,647 nm. The sizing model for FEA uses an aluminum material for all panels. The layout definition leads to 438 thickness design variables. Two meshes of 13,326 and 20,825 quadrilaterals are used for the alternating projection method. For subsonic concepts, aeroelastic analysis never failed, so there is no need to scale Young's modulus. After setting safety factor $=1.67$, $\mathrm{min}$ fuselage gauge $=0.15$ inches, and min wing gauge $=0.12$ inches, the FEA-based fuselage and wing weights match FLOPS fuselage and wing weights with relative errors less than $1.0 \%$ and $1.2 \%$, respectively (cf. Fig. 18). For this sizing problem, all weights generated during alternating projection iterations differ by less than $5 \%$; so the alternating projection iteration is not necessary.

\begin{tabular}{|c|c|c|}
\hline G450-like & Fuselage & Wing \\
\hline 13,326 Quads & $5,836 \mathrm{lbs}$ & $5,100 \mathrm{lbs}$ \\
\hline 20,825 Quads & $5,838 \mathrm{lbs}$ & $5,144 \mathrm{lbs}$ \\
\hline FLOPS Weights & $5,883 \mathrm{lbs}$ & $5,158 \mathrm{lbs}$ \\
$\begin{array}{c}\text { Span }=67.5 \mathrm{ft} \\
\text { Length }=79.6 \mathrm{ft}\end{array}$ \\
\hline
\end{tabular}

Fig. 18 Replication of FLOPS weights for G450-like concept using mesh-independent solutions.

American Institute of Aeronautics and Astronautics 
The max displacements for two FEA solutions at $2.5 \mathrm{G}$ trim condition are about $1 \mathrm{ft}$ even though no displacement constraint is used in sizing. Based on safety factor of 1.67 for the FEA-based weights, FLOPS fuselage and wing weights are considered to be credible, which should be the case because FLOPS weight regression formulas are derived from the conventional aircraft data and the G450-like concept is a conventional vehicle.

\section{E. B737-like Subsonic Concept}

The last example is a single-aisle commercial transport similar to a Boeing 737-800. The mission and payload are similar to 737-800, but some changes have been made to prepare it to be an advanced hybrid-electric aircraft. This concept has a cruise Mach of 0.785 , a cruise altitude of 43,000 ft, a cruise weight of 150,000 lbs, a ZFW of 115,287 $\mathrm{lbs}$, and a range of $3,500 \mathrm{~nm}$. The sizing model for FEA uses an aluminum material for all panels. The layout definition leads to 359 thickness design variables. Two meshes of 17,273 and 38,131 quadrilaterals are used for the alternating projection method. For subsonic concepts, aeroelastic analysis never failed, so there is no need to scale Young's modulus. After setting safety factor $=1.95$, min fuselage gauge $=0.13$ inches, and min wing gauge $=0.18$ inches, the FEA-based fuselage and wing weights match FLOPS fuselage and wing weights with relative errors less than $1.3 \%$ and $1.0 \%$, respectively (cf. Fig. 19). Unlike the G450-like case, the alternating projection iteration is necessary for sizing the B737-like concept (cf. Fig. 20).

\begin{tabular}{|c|c|c|}
\hline B737-like & Fuselage & Wing \\
\hline 17,273 Quads & $16,544 \mathrm{lbs}$ & $15,143 \mathrm{lbs}$ \\
\hline 38,131 Quads & $16,619 \mathrm{lbs}$ & $15,213 \mathrm{lbs}$ \\
\hline FLOPS Weights & $16,747 \mathrm{lbs}$ & $15,148 \mathrm{lbs}$ \\
\hline
\end{tabular}

Fig. 19 Replication of FLOPS weights for B737-like concept using mesh-independent solutions.

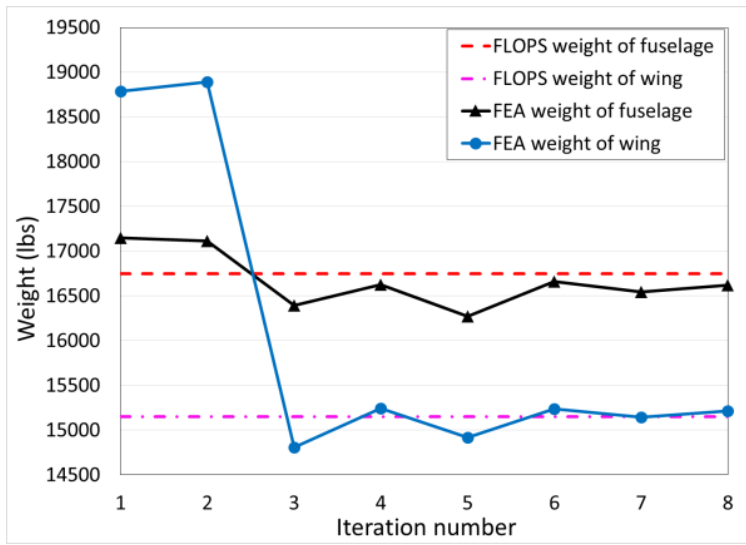

Fig. 20 Alternating projection iteration for B737-like concept to replicate FLOPS weights.

The max displacements for two FEA solutions at $2.5 \mathrm{G}$ trim condition are about $7.7 \mathrm{ft}$. Based on safety factor of 1.95 for the FEA-based weights, FLOPS fuselage and wing weights for the B737-like concept are considered to be credible. This should be expected because Boeing 737 aircraft weight data were used in development of FLOPS weight regression formulas.

\section{Concluding Remarks}

A robust automation process from an OpenVSP geometry to a PATRAN plot of the static aeroelastic solution of sized structure is developed for any aircraft wing-body configuration. The automation process is called ConceptFEA. ModelCenter is used for the current implementation of ConceptFEA. Three supersonic and two subsonic aircraft concepts are used to demonstrate ConceptFEA as a physics-based weight estimation tool.

Automated mesh generation using FEM-ready geometry and the PATRAN meshing code Paver is extremely robust. Multiple meshes are generated for each concept. Mesh-independent fuselage and wing weight estimates are obtained for all five concepts. For each of the five concepts, by using proper sizing constraints, ConceptFEA can match [calibrated] FLOPS fuselage and wing weights accurately (relative errors $<3.5 \%$ ). For three of the five aircraft concepts - one subsonic and two supersonic concepts - it is necessary to use the alternating projection for mesh-

American Institute of Aeronautics and Astronautics 
independent sizing solutions. Validity of FLOPS structural weights can be determined by the resulting sizing constraints for matching FLOPS weights. For example, the safety factor for sizing of the NASA N+2 concept had to be lower than the FAA standard value to match FLOPS weights, so FLOPS weights for the NASA N+2 could be questionable. If the sizing constraints and isotropic material properties are known, ConceptFEA can generate FEAbased fuselage and wing weights to correct FLOPS weights.

A variety of automation tools are developed for ConceptFEA; a sizing model for a new concept can be easily set up in a few hours once FLOPS weight and mission data are available. This is a significant reduction in the turn-around time compared to the many months previously required to perform FEA of a new concept. The substantial reduction in the required analysis time enables systems analysts to perform physics-based weight estimation for conceptual design of revolutionary aircraft configurations. The benefit of replacing the current empirical-based methods with physics-based weight estimation is more reliable system-level trade studies for better technology investment decisionmaking.

\section{Acknowledgments}

This work is funded by NASA Commercial Supersonic Technology Project and NASA Transformational Tools \& Technologies Project. The authors also want to thank James Fenbert for providing innovative and intuitive visual images showing the complicated relationships between different NASTRAN data entities.

\section{References}

[1] Li, W., and Robinson, J., “Automated Generation of Finite-Element Meshes for Aircraft Conceptual Design,” AIAA Paper 2016-3287, June 2016.

[2] NASTRAN, Version 2016.0, MSC Software, URL:http://www.mscsoftware.com/product/msc-nastran [retrieved 9 July 2017].

[3] McCuller, L., "FLOPS User Guide,” NASA Langley Research Center, Hampton, Virginia, 2008.

[4] ModelCenter, Version 11.2, Phoenix Integration Inc., URL:http://www.phoenix-int.com [retrieved 9 July 2017].

[5] OpenVSP, Version 3.11.0, Open Source, URL:http://www.openvsp.org [retrieved 9 July 2017].

[6] PATRAN, Version 2012.2 64-Bit, MSC Software, URL:http://www.mscsoftware.com/product/patran [retrieved 9 July 2017].

[7] Ardema, M., Chambers, M., Patron, A., Hahn, A., Miura, H., and Moore, M., "Analytical Fuselage and Wing Weight Estimation of Transport Aircraft," Tech. Report NASA TM-110392, May 1996.

[8] Laughlin, T., Corman, J., and Mavris, D., "A Parametric and Physics-Based Approach to Structural Weight Estimation of the Hybrid Wing Body Aircraft,” AIAA Paper 2013-1082, January 2013.

[9] Beltramo, M., Trapp, D., Kimoto, B., and Marsh, D., "Parametric Study of Transport Aircraft Systems Cost and Weight," NASA CR151970, April 1977.

[10] Wells, D., Horvath, B., and McCullers, L., “The Flight Optimization System Weights Estimation Method,” NASA TM 2017219627, Volume 1, January 2017.

[11] Silva, W., Garza, A., Zink, S., Bounajem, E., Johnson, J., Buonanno, M., Sanetrik, M., Chwalowski, P., Yoo, S., and Hur, J., "An Overview of the NASA High Speed ASE Project: Aeroelastic Analysis of a Low-Boom Supersonic Configuration," AIAA Paper 2015-0684, January 2015.

[12] NASTRAN, “High Performance Computing User's Guide,” MSC Software Corporation, November 2016.

[13] Morgenstern, J., Buonanno, M., Yao, J., Murugappan, M., Paliath, U., Cheung, L., Malcevic, I., Ramakrishnan, K., Pastouchenko, N., Wood, T., Martens, S., Viars, P., Tersmette, T., Lee, J., Simmons, R., and Plybon, D., Alonso, J., Palacios, F., Lukaczyk, T., and Carrier, G., "Advanced Concept Studies for Supersonic Commercial Transports Entering Service in the 2018-2020 Period Phase 2,” NASA CR-2015-218719, NASA, July 2015.

American Institute of Aeronautics and Astronautics 